\title{
Processing of temporal duration information in working memory after frontodorsal tumour excisions
}

\author{
Thomas D. Hälbig, ${ }^{\mathrm{a}, 1}$ D. Yves von Cramon, ${ }^{\mathrm{a}}$ Urs D. Schmid,,${ }^{\mathrm{b}, 2}$ \\ Claudius Gall, ${ }^{\mathrm{b}}$ and Angela D. Friederici ${ }^{\mathrm{a}, *}$ \\ ${ }^{a}$ Max-Planck-Institute of Cognitive Neuroscience, Stefanstraße 1a, Leipzig, D-04103, Germany \\ ${ }^{\mathrm{b}}$ Department of Neurosurgery, Ludwig-Maximilians University, Munich, Germany
}

Accepted 24 June 2002

\begin{abstract}
This study aimed to test the hypothesis that impairments of temporal duration processing after frontal lobe lesions reflect deficits in executive monitoring functions rather than a domain-specific deficit in the maintenance of duration information in working memory. Patients with frontodorsal lesions, clinical controls with post-central lesions, and healthy controls performed recognition and classification tasks, which should allow for testing maintenance and monitoring functions, respectively. Results showed mild non-selective impairments of the frontal patients on both temporal and spatial recognition tasks, but a marked selective degradation on temporal classification while performance on spatial classification was unimpaired. This suggests that maintenance of duration information in working memory after frontal lesions is basically preserved but that, depending on executive task characteristics, there is a specific deficit in the strategic organization of this type of information.
\end{abstract}

(c) 2002 Elsevier Science (USA). All rights reserved.

Keywords: Frontal lobe; Temporal information; Spatial information; Maintenance; Strategic processing

\section{Introduction}

The notion of working memory used here refers to the temporal activation of neuronal structures mediating the storage and manipulation of information necessary for a large variety of cognitive tasks. A number of studies suggest that, in working memory, different types of information, such as spatial and object information, are processed by different modules, i.e., by functionally domain-specific and neuroarchitectonically distinct systems (Farah, Hammond, Levine, \& Calvanio, 1988; Mecklinger \& Pfeifer, 1996; Newcombe, Ratcliff, \& Damasio, 1987; Ruchkin, Johnson, Grafman, Canoune, \& Ritter, 1997; Smith et al., 1995; Tresch, Sinnamon,

\footnotetext{
${ }^{*}$ Corresponding author. Fax: +49-341-9940-113.

E-mail address: angelafr@cns.mpg.de (A.D. Friederici).

${ }^{1}$ Present address: Department of Neurology, Charité, Berlin, Germany.

${ }^{2}$ Present address: Neurosurgical Unit, Klinik im Park, Zürich, Switzerland.
} 
\& Seamon, 1993; Ungerleider \& Mishkin, 1982; Wilson, O'Scalaidhe, \& GoldmanRakic, 1993). In contrast, it is less clear, whether the modularity assumption holds for the processing of temporal information as well.

Temporal information to be encoded in memory has two dimensions-temporal order and temporal duration information. Temporal duration refers to the storage of the persistence of a given event or the interval between two successive events; temporal order refers to the storage of the sequential occurrence of events (Nichelli, 1993). In this paper, we will focus primarily on temporal duration, although there is evidence that the processing of order and duration information might be related (Boltz, 1993; Miller \& Barnet, 1993).

Within the framework of internal clock models (Church, 1989; Gibbon, Church, \& Meck, 1984; Treisman, Faulkner, Naish, \& Brogan, 1990; Zakay \& Block, 1996), which describe formal properties of the cognitive processes underlying duration processing, it is assumed that different components contribute each to a specific functional level of duration processing. Fundamental to this is the hypothesis that clock-counter mechanisms monitor the passage of subjective time (clock stage). Records of accumulated time are transferred to working or reference memory (memory stage) for later comparison (decision stage). The decision stage compares current time with remembered time and identifies an appropriate response outcome.

Electrophysiological, neuropharmacological, and lesion data, as well as imaging studies, have indicated that the neural correlate mediating duration processing consists of a network of distributed neural systems, involving cerebellum (Casini \& Ivry, 1999; Ivry \& Keele, 1989; Jueptner et al., 1995; Nichelli, Alway, \& Grafman, 1996), basal ganglia (Gibbon, Malapani, Dale, Corby, \& Gallistel, 1997; Hinton, Meck, \& MacFall, 1996; Meck, 1996; Pastor, Artieda, Jahanshahi, \& Obeso, 1992; Rammsayer, 1994; Rammsayer \& Lima, 1990), parietal (Cabeza et al., 1997; Coull \& Nobre, 1998), and prefrontal cortex (Casini \& Ivry, 1999; Gibbon et al., 1997; Hinton et al., 1996; Nichelli, Clark, Hollnagel, \& Grafman, 1995). Yet, for each of these structures there is little agreement on their functional contribution to duration processing and on their involvement in the processing of different time ranges (Gibbon et al., 1997; Ivry, 1996; Ivry \& Keele, 1989; Jueptner et al., 1995; Meck, 1996; Nichelli et al., 1995; Rammsayer, 1994). The precise functional role of the frontal cortex in particular is a subject of considerable controversy (Gibbon et al., 1997). Frontal lobe function has been brought in touch in different theories with clock or counter mechanisms (Grafman, 1989; Miall, 1996; Niki \& Watanabe, 1979) as well as with the memory stage (Mangels, Ivry, \& Shimizu, 1998; Nichelli et al., 1995).

Studies with brain damaged patients provided evidence for prefrontal cortical involvement in the processing of temporal information in memory (Kesner, Hopkins, \& Fineman, 1994; Mangels, 1997; Mangels et al., 1998; McAndrews \& Milner, 1991; Milner, Corsi, \& Leonard, 1991; Nichelli et al., 1995; Shimamura, Janowsky, \& Squire, 1990). Regarding temporal order, several studies indicated a dissociation of temporal order and item memory (Cabeza et al., 1997; Kesner et al., 1994; McAndrews \& Milner, 1991; Milner et al., 1991; Shimamura et al., 1990). For example, the importance of the frontal lobes for temporal order memory is supported by studies showing that patients with frontal lobe lesions exhibit deficits in tests for serial order reconstruction (Shimamura et al., 1990) and relative recency judgments (Milner et al., 1991). As compared to other amnesics, patients with Korsakoff syndrome, who suffer from frontal lobe atrophy (Shimamura, Jernigan, \& Squire, 1988) exhibit not only impairment of item memory but also a disproportional deficit on tests of temporal order memory.

Concerning the processing of duration information, recent electrophysiological (Casini \& Macar, 1996; Elbert, Ulrich, Rockstroh, \& Lutzenberger, 1991; Schubotz \& Friederici, 1997) and lesions studies (Casini \& Ivry, 1999; Mangels et al., 1998; 
Nichelli et al., 1995) have also emphasized the involvement of frontal brain structures. For example, in a study using event-related brain potentials (ERP), differently distributed ERPs were reported for temporal duration and spatial location working memory tasks with stimulus durations in the millisecond range $(275,300,570$, $650 \mathrm{~ms}$ ). There was a frontodorsal focus on temporal tasks and a parieto-occipital focus on spatial tasks (Schubotz \& Friederici, 1997). Compatible with these results, we provided evidence for the functional dissociation of temporal duration and spatial location working memory in intact young adults using a selective interference paradigm (Hälbig, Mecklinger, Schriefers, \& Friederici, 1998). Lesion studies support the notion that the frontal cortex is involved in duration processing (Casini \& Ivry, 1999; Mangels et al., 1998; Nichelli et al., 1995). Using a bisection procedure, Nichelli et al. (1995) found impaired accuracy on duration discrimination in the millisecond range (100-900 ms) and for longer intervals (8-32 s), but normal performance on a line-length discrimination task.

With respect to other domains, such as spatial and object processing, it has been proposed that different parts of the prefrontal cortex make distinct contributions to working memory operations, depending on the informational domain (GoldmanRakic, 1994; Wilson et al., 1993; Levy \& Goldman-Rakic, 2000). Its domain-specific components are supposed to be parts of functional networks, each interconnected with domain-relevant long-term storing sites in posterior cortical regions. According to this view, domain-specific prefrontal substructures are involved in the activation and monitoring of information. Considering duration as a stimulus dimension (equivalent to shape and location), one could be tempted to suppose domain-specific neural assemblies contributing to duration processing. However, even if there is considerable evidence that memory for temporal duration depends on the integrity of the frontal lobes (Casini \& Ivry, 1999; Casini \& Macar, 1996; Elbert et al., 1991; Mangels et al., 1998; Nichelli et al., 1995; Schubotz \& Friederici, 1997), variability of lesion size and location in the cited neuropsychological studies do not allow to identify any specific area within the frontal cortex that is involved necessarily in the processing of temporal duration information.

Alternatively, another model of prefrontal organization (D'Esposito et al., 1998; Owen et al., 1998; Petrides, 1996; Postle, Berger, \& D'Esposito, 1999) suggests a subdivision of the prefrontal cortex depending rather upon the type of processing than on the informational domain. According to this model, monitoring and manipulation of information within working memory depend on the mid-dorsolateral frontal cortex, whereas maintenance of information involves the mid-ventrolateral and -dorsolateral frontal cortex (Postle et al., 1999). It is well established that patients with frontal lobe lesions exhibit deficits in functions related to the central executive such as attention allocation, inhibition of task irrelevant information, set-shifting, strategy use, etc. (Baddeley, 1986; Fuster, 1989; Smith et al., 1995). Given the involvement of the frontal lobes in executive monitoring functions, several authors propose that deficits in temporal memory might be secondary to executive dysfunctions (Casini \& Ivry, 1999; Mangels, 1997; Mangels et al., 1998; Shimamura et al., 1990; Tracey, Faro, Mohamed, Pinsk, \& Pinus, 2000).

Consistent with the latter interpretation, Korsakoff patients show not only degraded temporal order memory, but also demonstrate poor performance in learning and applying rules as indicated by their scores in the Wisconsin Card Sort Test (Shimamura et al., 1988), and failure to exhibit release from proactive interference (Squire, 1982). Moreover, as reported recently (Mangels, 1997), memory deficits for temporal order in frontal lobe patients occur only under intentional and not incidental learning conditions. This suggests that the patients are able to form codes for temporal information, but seem to have a specific deficit in the strategic organization of this type of information during encoding and retrieval. 
A study by Casini et al. (1999) supports the hypothesis that defective temporal processing after frontal lobe lesions is related to executive deficits. When subjects had to make concurrent judgments on duration and frequency information, frontal lobe patients were impaired on both tasks, whereas cerebellar patients showed increased response variability only on temporal tasks. Furthermore, Mangels et al. (1998) found in a series of experiments that defective duration discrimination in frontal lobe patients depends on the range of tested durations. Robust deficits were reported only for long intervals ( $4 \mathrm{~s})$. These deficits, however, appeared to be non-selective, as the frontal lobe patients also exhibited working memory deficits on frequency discrimination tasks.

Since deficits in temporal processing after frontal lobe lesions appear to depend on strategic task demands (Mangels, 1997; Nichelli, 1993; Shimamura et al., 1990), the observed duration deficits are presumably not related to a disruption of domainspecific neural assemblies. It is conceivable that defective temporal processing is secondary to defective strategic monitoring functions (Mangels, 1997; Mangels et al., 1998; Petrides, 1996; Shimamura et al., 1990).

The aim of the present study was to examine more specifically the hypothesis that deficits in the processing of duration information in working memory after frontal lobe lesions reflect a deficit in the strategic organization of information. Using a lesion data approach, we compared temporal working memory in patients with frontodorsal lesions restricted to the superior or middle frontal gyri, and in patients with post-central lesions who served as clinical control subjects relative to intact subjects.

To assess the possibly differential contribution of the frontal lobes in maintenance and strategic monitoring, the three groups were each tested on recognition and classification working memory tasks. The tasks were embodied in a clinically adapted version of a dual-task paradigm used in a previous study (Hälbig et al., 1998). Similar to other recent lesion studies on temporal processing (Casini \& Ivry, 1999; Mangels et al., 1998; Nichelli et al., 1995). working memory was tested for durations in the millisecond range. Both the recognition and the classification tests had encoding and storage components in common. They differed, however, regarding strategic task characteristics and retention intervals, with the classification tasks placing additional demands on strategic relational processing and the recognition tasks placing additional demands by rather long retention intervals. Based on recent evidence for the functional separability of temporal duration and spatial information memories (Hälbig et al., 1998; Schubotz \& Friederici, 1997) there was a corresponding spatial working memory condition, for each type of temporal task. This should have allowed us to control for possible effects of the nature of stimulus material as compared to the processing characteristics of the task.

In the recognition memory tasks, subjects were required to indicate whether a repeatedly presented visual stimulus was same or different in duration (temporal recognition) or in location (spatial recognition). In the temporal task, the stimulus was presented on all trials at the same spatial position, whereas presentation durations varied. In the spatial task, the same stimulus was shown on all trials for the same duration, whereas spatial positions varied. The recognition tasks demanded encoding of the presentation duration of a reference stimulus during the study phase, maintenance across delay and during presentation of the test stimulus and a comparison of reference and test stimulus resulting in a binary "same-different" decision.

In the classification tasks, a cross and a star were shown successively in random order. Subjects had to indicate whether the star was longer in presentation duration (temporal classification) or higher in position (spatial classification) than the cross. As in the recognition tasks, the stimuli in temporal classification were shown over all 
trials at the same spatial position, while the presentation duration was fixed for spatial classification. In comparison to the recognition task, the classification tasks required more than the comparison of one item with another in memory. First, during stimulus encoding, the crucial item information (i.e., duration or location) had to be associated with the different object characteristics (that is, star or cross). Furthermore, since the reference stimulus (star) appeared in half of the trials temporally before the test stimulus (cross) and in the other half after the test stimulus, the crucial item information had to be placed in a temporal context. Thus, the classification task demanded not only attentional resources to form internal representations of the crucial stimulus feature (i.e., temporal duration and spatial position), but sustained attention as well to the varying stimulus context characteristics.

Lesions in our frontal patients were restricted to the superior and middle frontal gyri. Thus, they affected mid-dorsolateral areas of the prefrontal cortex, which are supposed to mediate executive monitoring, irrespective of the type of information (D’Esposito et al., 1998; Owen et al., 1998; Petrides, 1996). We therefore predicted a higher degree of impairment on temporal classification tasks as compared to temporal recognition tasks.

If deficits in temporal memory after frontal lesions are secondary to executive dysfunctions, and thus reflect a supramodal deficit (Mangels, 1997; Mangels et al., 1998; Shimamura et al., 1990), domain-specific deficits should not occur as long as process-specific parameters are held constant. Yet, acquisition and representation of temporal information in neural systems presumably differ fundamentally from the processing of object or spatial stimulus features. Representations of temporal duration are acquired dynamically, demanding attentional gating until stimulus off-set and thus are specifically sensitive to the use of effective encoding and attentional allocation strategies (Gilliland \& Martin, 1940; Kileen \& Weiss, 1987; Niki \& Watanabe, 1979; Zakay \& Block, 1996). Since the classification task demanded additional strategic monitoring of attentional resources, a higher degree of impairment in frontal lobe patients is predicted for temporal duration classification as compared to the spatial task.

Both types of classification and recognition tasks were combined in a dual-task design, with the classification tasks to be performed during the recognition tasks' retention intervals. Findings of a previous study (Hälbig et al., 1998) revealed that temporal and spatial working memory are subject to selective interference when concurrent tasks of the same informational domain are performed. Based on these findings for the temporal and spatial recognition tasks, we predicted selective interference produced by the temporal and spatial classification task respectively. Moreover, as far as the prefrontal cortex is specifically involved in temporal memory, frontal lobe patients selective interference effects on temporal recognition caused by concurrent temporal classification should be more pronounced than selective interference on spatial recognition.

\section{Method}

\subsection{Participants}

Patients were recruited from the clinical archives of the Departments of Neurosurgery of the Ludwig-Maximilians University of Munich and the Free University of Berlin. In the search for right-handed patients with well defined unilateral focal lesions, we chose patients whose lesions resulted as a consequence of surgical resection of a tumour. We excluded patients who were older than 55 years, those with multiple lesions or tumours of high malignancy and those receiving radiation therapy. 
Table 1

Clinical data of 14 patients participating in the study

\begin{tabular}{|c|c|c|c|c|c|}
\hline Patient & Sex & Age & $\begin{array}{l}\text { Lesion } \\
\text { side }\end{array}$ & Lesion location $^{\mathrm{a}}$ & Medical diagnosis \\
\hline \multicolumn{6}{|c|}{ Frontal group } \\
\hline PAT 01 & $\mathrm{f}$ & 48 & $\mathrm{~L}$ & $\mathrm{~F} 1^{\mathrm{c}}$ & Astrocytoma $^{\mathrm{b}}$ \\
\hline PAT 02 & $\mathrm{f}$ & 28 & $\mathrm{~L}$ & $\mathrm{~F} 1 / \mathrm{F} 2^{\mathrm{c}}$ & Astrocytoma $^{\mathrm{b}}$ \\
\hline PAT 03 & $\mathrm{f}$ & 38 & $\mathrm{R}$ & $\mathrm{F} 1 / \mathrm{F} 2^{\mathrm{c}}$ & Astrocytoma ${ }^{b}$ \\
\hline PAT 04 & $\mathrm{f}$ & 32 & $\mathrm{R}$ & $\mathrm{F} 1^{\mathrm{c}}$ & $\begin{array}{l}\text { Cavernous } \\
\text { malformation }\end{array}$ \\
\hline PAT 05 & $\mathrm{f}$ & 34 & $\mathrm{~L}$ & $\mathrm{~F} 1 / \mathrm{F} 2^{\mathrm{c}}$ & Astrocytoma ${ }^{b}$ \\
\hline PAT 06 & $\mathrm{f}$ & 41 & $\mathrm{R}$ & $\mathrm{F} 1^{\mathrm{c}}$ & Astrocytoma $^{\mathrm{b}}$ \\
\hline PAT 09 & $\mathrm{~m}$ & 37 & $\mathrm{R}$ & $\mathrm{F} 1^{\mathrm{c}}$ & $\begin{array}{l}\text { Cavernous } \\
\text { malformation }\end{array}$ \\
\hline PAT 14 & $\mathrm{~m}$ & 52 & $\mathrm{R}$ & $\mathrm{F} 1 / \mathrm{F} 2^{\mathrm{c}}$ & Astrocytoma $^{\mathrm{b}}$ \\
\hline PAT 18 & $\mathrm{f}$ & 33 & $\mathrm{~L}$ & $\mathrm{~F} 1^{\mathrm{c}}$ & Astrocytoma ${ }^{b}$ \\
\hline \multicolumn{6}{|c|}{ Clinical control group } \\
\hline PAT 07 & $\mathrm{f}$ & 28 & $\mathrm{R}$ & $\begin{array}{l}\text { Paramedian portion } \\
\text { of the post-central gyrus } \\
\text { and adjacent superior } \\
\text { parietal lobule }\end{array}$ & Astrocytoma $^{\mathrm{b}}$ \\
\hline PAT 10 & $\mathrm{f}$ & 35 & $\mathrm{~L}$ & $\begin{array}{l}\text { Inferior portion of the } \\
\text { post-central gyrus }\end{array}$ & Astrocytoma $^{\mathrm{b}}$ \\
\hline PAT 13 & $\mathrm{f}$ & 48 & $\mathrm{R}$ & $\begin{array}{l}\text { Post-central gyrus and } \\
\text { minor portion of the } \\
\text { adjacent precentral } \\
\text { gyrus }\end{array}$ & $\begin{array}{l}\text { Cavernous } \\
\text { malformation }\end{array}$ \\
\hline PAT 15 & f & 34 & $\mathrm{R}$ & $\begin{array}{l}\text { Paramedian of the } \\
\text { pre- and post-central } \\
\text { gyri, extended white } \\
\text { matter lesions due to } \\
\text { perifocal edema }\end{array}$ & Astrocytoma ${ }^{b}$ \\
\hline PAT 16 & $\mathrm{f}$ & 32 & $\mathrm{~L}$ & $\begin{array}{l}\text { Gyrus supramarginalis, } \\
\text { gyrus angularis, and } \\
\text { posterior portion of the } \\
\text { superior temporal gyrus }\end{array}$ & Astrocytoma $^{\mathrm{b}}$ \\
\hline
\end{tabular}

${ }^{a}$ F1: Superior frontal gyrus; F2: Middle frontal gyrus.

${ }^{\mathrm{b}}$ WHO 11 .

${ }^{\mathrm{c}}$ See Fig. 1.

Patients who showed visual field defects or who had signs of visual neglect were also excluded. Table 1 represents a summary of the relevant clinical data on both the frontal and the clinical control patients who were chosen for participation in the study. All patients gave informed consent after the nature of the procedure had been fully explained.

The final experimental group of frontal patients included nine subjects with frontal lesions, all affecting the superior or middle frontal gyri (Table 1). Five patients with post-central lesions were selected as clinical control subjects (Table 1). Ten paid volunteers matched for age, educational level and handedness served as controls. The frontal patients' mean age was 38.1 years (SD 7.77), whereas that of the clinical controls was 35.4 years (SD 7.54). and that of the healthy controls 36.1 years (SD 7.48). Participants of each group had a mean of 12.4 (SD 1.34) years of education.

All but one participant (PAT 03) were free of any neurological symptoms at the time of the study. PAT 03 exhibited mild inferior paresis. Five of the patients were receiving anticonvulsant medication (PAT 01, 06, 14, 15, 16). The frontal patients were studied on average 28.8 months (SD 16.0) whereas the clinical control patients were tested on average 28.4 months (SD 13.97) after surgery. 
For the 14 patients who participated in the study, magnetic resonance imaging (MRI) was performed to determine lesion size and location at the time of the study. Fig. 1 shows images of the lesioned area in each of the frontal patients.

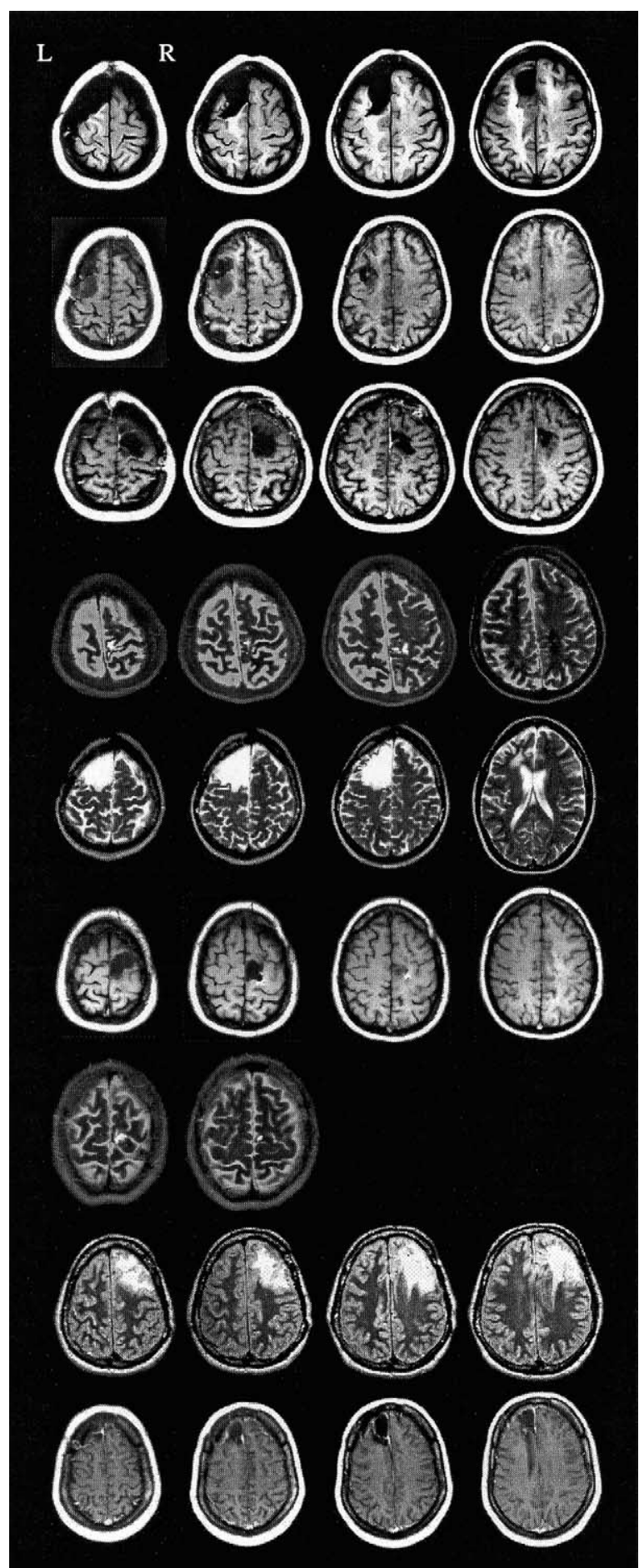

Fig. 1. Magnetic resonance images (MRI) of the lesioned area in each of the frontal patients. Image order from top to bottom corresponds to patient order as presented in Table 1. Note that for PATs 01, 02, 03, 06, 18, axial $\mathrm{T}_{1}$-weighted images are presented, whereas for PATs $04,05,09,14$, axial $\mathrm{T}_{2}$-weighted images are shown. 
Temporal Recognition Task

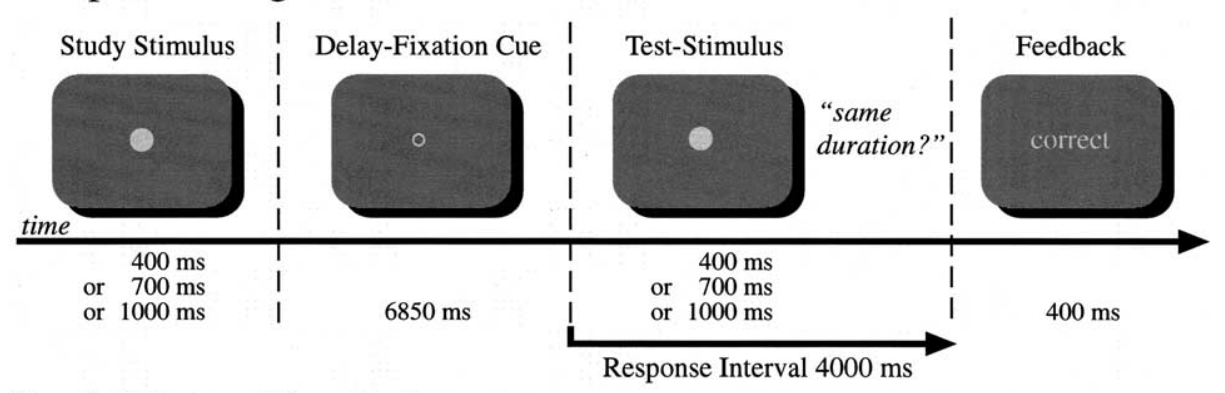

\section{Spatial Recognition Task}

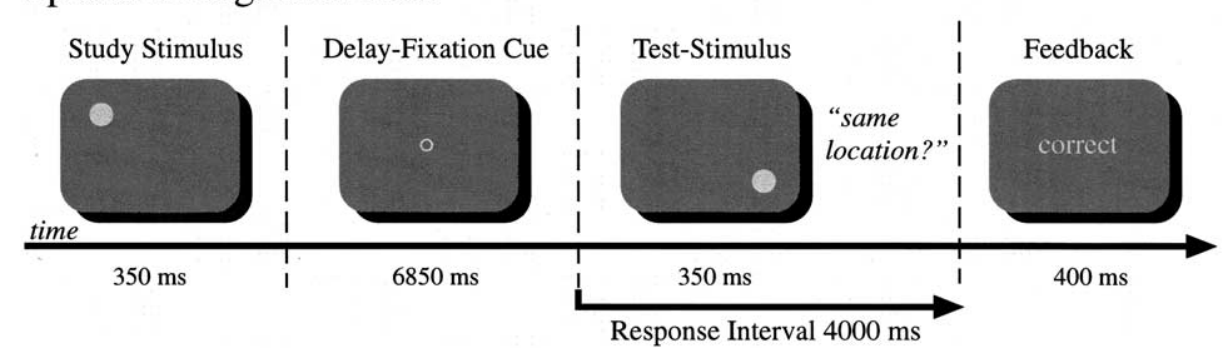

Fig. 2. Schematic drawing of the temporal and spatial recognition tasks. In all temporal tasks the stimuli were presented in the center of the screen whereas presentation duration varied randomly $(400,700$, or $1000 \mathrm{~ms}$ ). The stimulus position in the spatial tasks, in comparison, varied randomly, whereas presentation duration was fixed $(350 \mathrm{~ms})$.

\subsection{Experimental design}

\subsubsection{Recognition tasks}

In the study phase of the temporal recognition task (Fig. 2), a dot was presented in the center of the screen with a duration of either 400,700 , or $1000 \mathrm{~ms}$. The subjects were asked to memorize this duration. During the basic-delay that amounted to $6850 \mathrm{~ms}$, a fixation cue was shown in the center of the screen and subjects merely had to wait for the upcoming test stimulus. After the retention interval, the dot was presented again with a duration of either 400,700 , or $1000 \mathrm{~ms}$. Presentation duration was either identical to the study phase duration (with a probability of .50) or not. The subjects' task was to press one of two response buttons when the duration was the same as in the study phase (old trials), and the other when it was not (new trials). Responses were required within a $4700 \mathrm{~ms}$ interval from stimulus onset and the subjects were provided with feedback (Correct, False, No response) that remained on the screen for $400 \mathrm{~ms}$.

Duration discrimination of a test stimulus against reference stimulus differs from old-new binary decisions on spatial or object features concerning experimental timing characteristics. In contrast to old-new decisions in the spatial tasks, the decision in old-trials in the temporal tasks is not possible before the presentation of the entire duration of the test stimulus, and in new-trials not before presentation of at least the duration that corresponds to the duration of the shorter of both the study and the test stimulus. Thus, in our tasks, the effective response intervals amounted counterbalanced to 3700,4000 , and $4300 \mathrm{~ms}$, respectively.

The same dot was shown in the spatial recognition task (Fig. 2) with $350 \mathrm{~ms}$ duration at one position of a $5 \times 5$ non-visible grid (side lines $15 \mathrm{~cm}$ ). The subjects' task was to memorize the position of the dot. After a retention interval of the same length as in the temporal primary task, the same dot was shown again at one of the 25 grid positions. The dot position was either identical to the study phase position (with a 


\section{Temporal Classification Task}

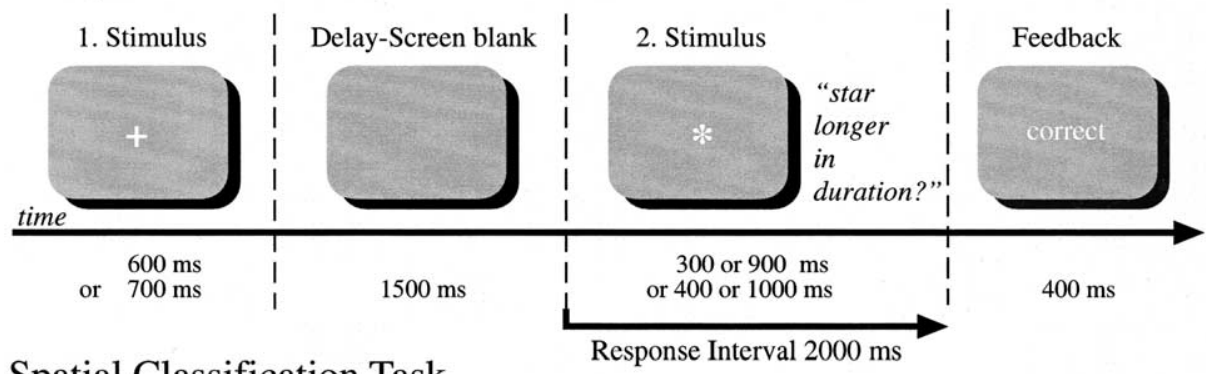

Spatial Classification Task

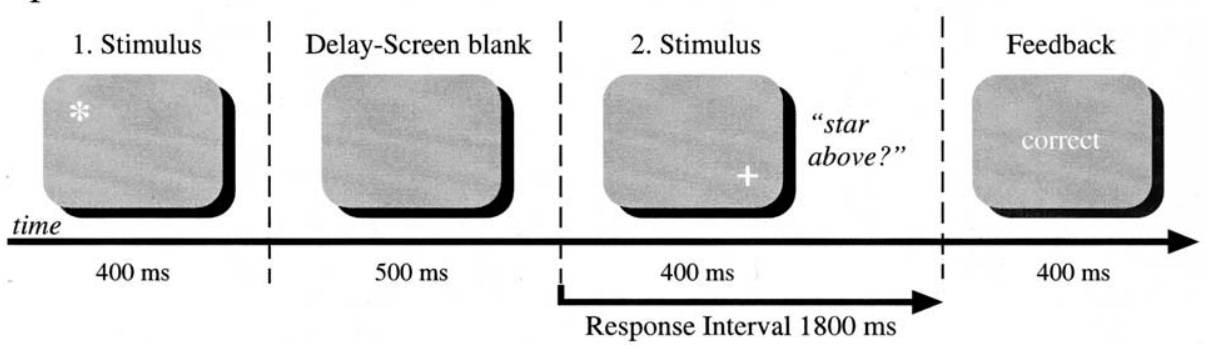

Fig. 3. Schematic drawing of the temporal and spatial classification tasks. In all temporal tasks the stimuli were presented in the center of the screen whereas presentation duration varied randomly (600 or $700 \mathrm{~ms}$ for the first stimulus and $300,400,900$, or $1000 \mathrm{~ms}$ for the second stimulus). The stimulus position in the spatial tasks, in comparison, varied randomly, whereas presentation duration was fixed $(400 \mathrm{~ms})$.

probability of .50) (old trials) or not (new trials). Responses had to be given within $4000 \mathrm{~ms}$ interval from stimulus onset. Feedback presentation was the same as in the temporal memory task.

\subsubsection{Classification tasks}

In the temporal classification task (Fig. 3), a cross and a star were shown successively in random order in the center of the screen. The ISI amounted to $1500 \mathrm{~ms}$. The first stimulus was presented with a duration of 600 or $700 \mathrm{~ms}$. Presentation duration of the second stimulus was either $300 \mathrm{~ms}$ longer or shorter. Subjects were instructed to indicate whether the star was presented for a longer duration than the cross (with a probability of .50) or not and to respond by pressing a response button. A feedback stimulus (duration: $400 \mathrm{~ms}$ ) was given $2500 \mathrm{~ms}$ after the onset of the second test item. As in the temporal recognition tasks, classification after presentation of the second stimulus was not possible before presentation of at least the duration that corresponded to the duration of the shorter of both stimuli. Thus the effective duration of the response intervals in the temporal classification tasks was $1800,1900,2100$, and $2200 \mathrm{~ms}$, respectively.

For the spatial classification task (Fig. 3), the star and the cross were shown successively in random order for $400 \mathrm{~ms}$ with an ISI of $500 \mathrm{~ms}^{3}$ Both items were located at two of 16 different and equally spaced positions of a non visible grid (side lines $12.5 \mathrm{~cm}$ ). Subjects had to indicate whether the star was presented above the position of the cross (with a probability of .50) or not and received feedback $1800 \mathrm{~ms}$ after stimulus onset. Based on the different timing characteristics of the secondary

\footnotetext{
${ }^{3}$ Just as several previous lesion studies (Kesner et al., 1994; Milner et al., 1991; Shimamura et al., 1990) comparing temporal and item memory have had, results of a pilot study for the present experiment yielded the problem of designing temporal and spatial tasks of an equivalent level of difficulty. Thus the use of different ISIs in spatial and temporal classification tasks was a necessary compromise in order to adjust general difficulty in both task conditions.
} 
task stimuli, the primary task retention intervals amounted counterbalanced to 8000 and $8100 \mathrm{~ms}$ for temporal classification, $6100 \mathrm{~ms}$ for spatial classification, and $6850 \mathrm{~ms}$ for the basic delay condition.

\subsubsection{Recognition-classification dual-task schema}

Recognition and classification tasks were embodied in a dual-task design, with the classification tasks to be performed during the retention intervals of the recognition tasks (Fig. 4). Thus, for both, the spatial and the temporal recognition tasks, there were three types of secondary task conditions: temporal classification, spatial classification and a basic delay condition, where subjects merely had to wait for the upcoming recognition task stimulus. Secondary tasks were separated from the preceeding recognition study phase and the following recognition test phase by delays of 2000 and $1000 \mathrm{~ms}$, respectively, where the screen remained blank. Based on the different timing characteristics of the secondary task stimuli, the primary task retention intervals amounted counterbalanced to 8000 and $8100 \mathrm{~ms}$ for temporal classification, $6100 \mathrm{~ms}$ for spatial classification, and $6850 \mathrm{~ms}$ for the basic delay condition.

\subsubsection{Stimuli}

The presentation of the memory tasks was produced by a Pentium 90 computer with a $12^{\prime \prime}$ monitor. The onset of the recognition tasks was indicated by a light gray circle of $6 \mathrm{~mm}$ in diameter that appeared for $1500 \mathrm{~ms}$ in the center of the screen. A dot of 24 min diameter presented in light gray against a dark gray background was used for both recognition tasks. The cross and the star used in the classification tasks were $22 \mathrm{~mm}$ in diameter. The gray circle which indicated trial onset was used as delay-task fixation cue. To ensure that the recognition task phases and the classifi-

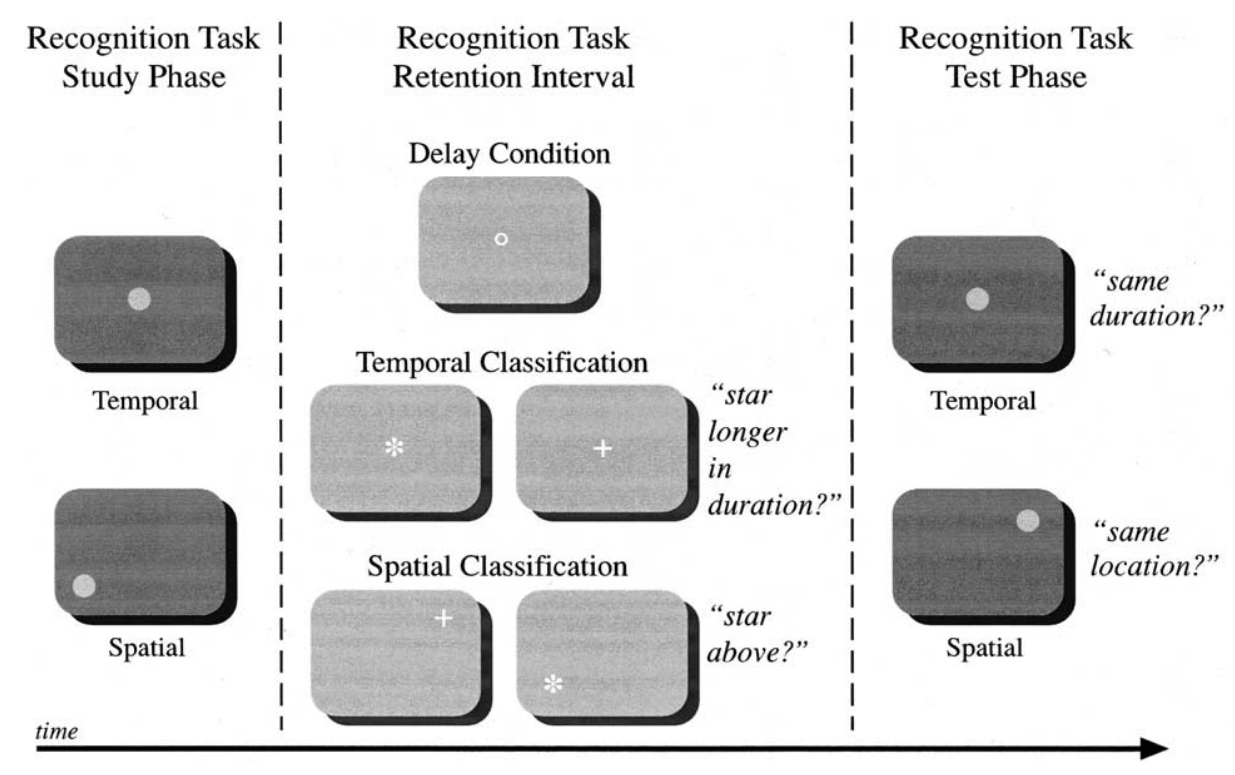

Fig. 4. Schematic drawing of the dual-task design comprised of temporal and spatial recognition tasks and three types of secondary tasks. Secondary tasks had to be performed during the retention interval of the recognition tasks. Thus, for both, the spatial and the temporal recognition tasks, there were three types of secondary task conditions: Temporal classification, spatial classification, and a basic delay condition, where subjects merely had to wait for the upcoming recognition task stimulus. The experimental design allowed the assessment of memory performance at different levels: Firstly, simple temporal and spatial recognition memory as tested by a recognition basic delay condition, further, temporal and spatial classification, and finally, performance on both, the temporal and spatial recognition tasks as a function of classification tasks. 
cation task could be clearly distinguished by the subjects, the classification task stimuli were presented in white on a light gray background.

\subsubsection{Procedure}

Subjects were tested individually in one experimental session lasting $75 \mathrm{~min}$. The session consisted of two parts separated by a break of $15 \mathrm{~min}$. Either the temporal or the spatial recognition task combined with the three different types of secondary tasks had to be performed in each part. The three types of trials within each part were presented as three blocks, beginning with the delay-task condition, followed by the incongruent classification task condition. Finally, the condition with the congruent classification task was presented. Blocks were separated by short breaks.

In each part, 48 trials were given in three blocks with 24 trials for the delay-task condition, and 12 trials each for the classification conditions which were used for statistical analyses. In addition, there were 4 practice trials at the beginning of each block. In order to prevent verbal receding of the three stimuli in the study phase of the temporal recognition task (e.g., short, medium, and long), there were 3 additional "catch" trials with stimulus durations of either 200 or $1200 \mathrm{~ms}$ in the study phases of each temporal recognition task block. To parallel the total trial number in both primary tasks, 3 trials were added to the spatial recognition task blocks.

The subjects were comfortably seated in a quiet and dimly lit room at a distance of $45 \mathrm{~cm}$ from the monitor and placed their thumbs on the response keyboard. The experimental sessions began with oral and written instruction. Subjects were told that accuracy was more important than response speed. On trials where they were uncertain about the correct response, subjects were instructed to make their "best estimate." Following the instruction there was a practice session that consisted of a shortened version of the experiment proper. Practice blocks were comprised of 12 trials.

\section{Results}

\subsection{Recognition task performance - delay-condition}

Fig. 5 displays accuracy data on the recognition tasks' delay-condition, where no concurrent classification task had to be performed, for each group separately. Two-

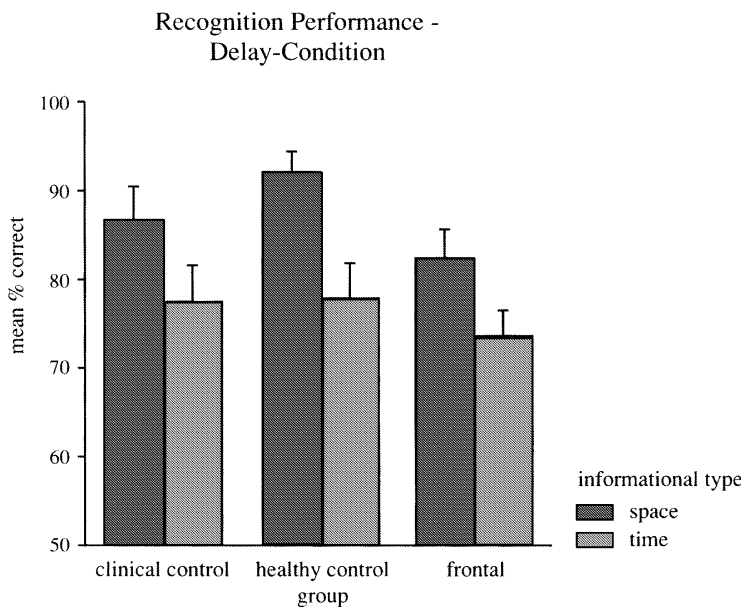

Fig. 5. Performance on spatial and temporal recognition tasks (delay condition) separately for clinical control group $(n=5)$, healthy control group $(n=10)$, and frontal group $(n=9)$. Bars represent mean accuracy and standard error of the means. 
way repeated measure ANOVAs with factors group (2 levels) and informational type ( 2 levels) were used to determine effects of prefrontal cortex damage on temporal and spatial memory relative to the healthy control group.

Results exhibit a significant effect of informational type $(F(1,17)=20.85$, $p<.0001$ ), indicating higher performance levels for spatial than for temporal recognition tasks. For the effect of group, the analysis revealed a mild trend but did not reach significance $(F(1,17)=3.57, p=.08)$. Thus, performance of the frontal group was slightly impaired as compared to the healthy control group. What is more important is that the analysis did not yield an interaction group $x$ informational type $(F(1,17)=1.15, p=.30)$, showing that there was no selective degradation for either the temporal or the spatial recognition task in frontal patients.

Due to smaller sample size, the Mann-Whitney $U$ test was used to determine effects of post-central cortical lesions on temporal and spatial recognition as compared to performance of healthy control subjects. Results did not show differences for both types of task (temporal: $U=28.5, p=.87$; spatial: $U=22.0, p=.43$ ), indicating that post-central lesions did not lead to significant degradation on either temporal or spatial recognition tasks.

\subsection{Recognition performance as function of classification task conditions}

Fig. 6 displays the mean accuracy for the temporal and spatial recognition tasks as a function of the three classification tasks separately for each group. Two-way repeated measure ANOVAs with factors recognition task (2 levels) and classification task ( 3 levels, including the basic delay condition) were used to determine interference effects produced by the three classification tasks on spatial and temporal recognition tasks. Interactions were assessed by planned $t$ tests for paired samples. For the clinical control group Wilcoxon's signed-rank test was used.

As apparent from Fig. 6, the overall performance level in the healthy control group was higher for the spatial than for the temporal recognition task $(F(1,9)=12.36$, $p<.007)$. As revealed by a significant effect of classification task $(F(2,18)=4.13$,

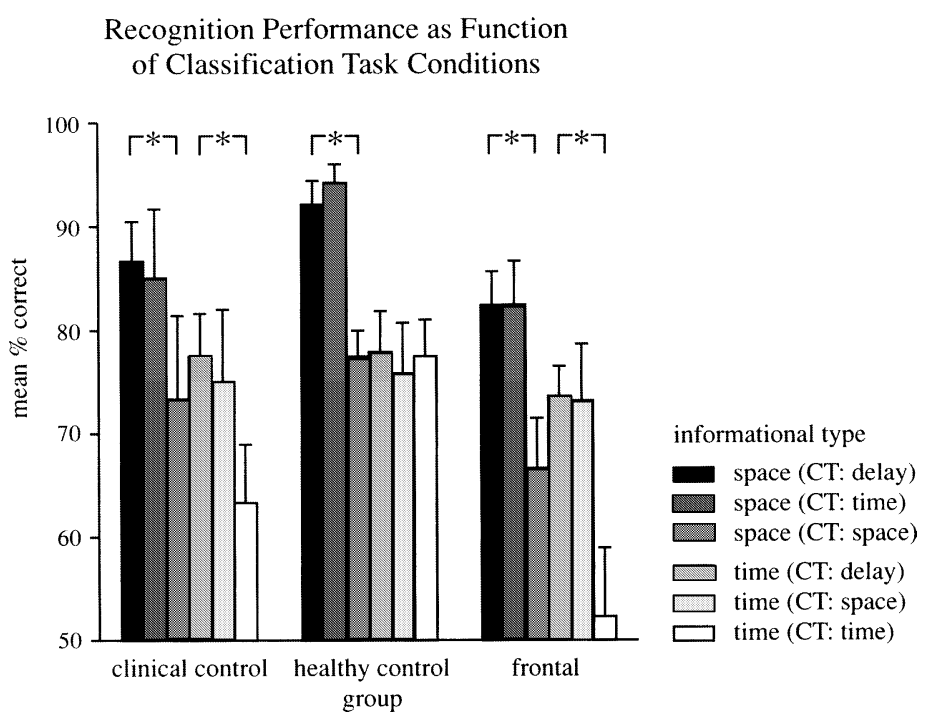

Fig. 6. Performance on spatial and temporal recognition tasks as a function of classification task (CT) conditions (delay, time, and space) separately for clinical control group $(n=5)$, healthy control group $(n=10)$, and frontal group $(n=9)$. Bars represent mean accuracy and standard error of the means. Asterisks denote significant differences between performance on the temporal or spatial recognition delay conditions and the temporal and spatial classification conditions for each group separately. 
$p=.03$ ), recognition task performance was differentially affected by the three classification tasks. Furthermore, a significant interaction recognition task $x$ classification task was obtained $(F(2,18)=7.34, p=.005)$. $t$ tests for paired samples revealed that spatial memory task performance was degraded by a spatial classification task as compared to the delay condition $(t(9)=4.02, p=.003)$ but not by a temporal classification task $(t(9)=.71, p=.49)$. In contrast, no interference effects were obtained for the temporal recognition tasks (delay $x$ spatial classification: $t(9)=.39$, $p=.70$; delay $x$ temporal classification $(t(9)=.12, p=.91)$. The latter result reflects that, under the temporal classification condition, performance was as good as under the delay condition. ${ }^{4}$

For the frontal group, the ANOVA revealed significant effects of recognition $(F(1,8)=6.24, p<.04)$ and classification task $(F(2,16)=4.42, p=.03)$ as well as an interaction $(F(2,16)=11.14, p<.001)$. Subsequent $t$ tests showed selective interference for both the spatial and the temporal recognition tasks. Temporal recognition task performance was degraded when the temporal classification task had to be performed as a secondary task $(t(8)=3.43, p<.01)$, but not when a spatial classification task served as a secondary task $(t(8)=.12, p=.91)$. Whereas spatial recognition task performance was degraded when a spatial classification task had to be performed as a secondary task $(t(8)=3.60, p<.01)$, but not when a temporal classification task served as a secondary task $(t(8)=.00, p>.99)$.

For the clinical control group, Wilcoxon's signed-rank test revealed that performance on the temporal recognition task was selectively degraded by a temporal classification task $(z=-1.99, p<.05)$, but not by a spatial classification task $(z=-.31, p>.05)$. Performance on the spatial recognition task was degraded $13 \%$ on average by a spatial classification task. However, presumably due to the high degree of variance of the relatively small sample $(n=5)$, statistical analysis did not reach significance $(z=-1.61, p=.11)$. In contrast, spatial performance was not degraded by a temporal classification task $(z=-.40, p=.69)$.

\subsection{Classification performance as function of recognition task conditions}

Before assessing classification task data with respect to differential effects of cortical lesions, we analyzed classification task performance of each group for selective interference effects possibly caused by the preceding recognition task study phases. Two-way repeated measure ANOVAs with factors recognition task (two levels) and classification task (two levels) were used for frontal and healthy control group each and, due to smaller sample size, the Wilcoxon signed-rank test for the clinical control group. Fig. 7 displays the percentage of correct classifications in the classification tasks as a function of the preceding recognition task study phases for each group separately.

\footnotetext{
${ }^{4}$ In the present study, healthy controls showed domain-specific interference only for the spatial domain. Their good performance on the temporal task contrasts with results of a previous study on young intact subjects (Hälbig et al., 1998). The failure to produce selective interference in both informational domains might reflect methodical differences between both studies. Note that, for the application of the experimental design in patients, modifications of task parameters were necessary. For example, we selected response intervals that were $2000 \mathrm{~ms}$ longer in duration than in the original version. Furthermore, in the present experiment, subjects were tested in one experimental session with a smaller number of trials per block. Finally, healthy subject groups in both studies differed with respect to education, sample size ( $n=24$ vs. $n=10$ in the present study), and mean age (26.3 years vs. 36.1 years in the present study). As revealed by descriptive single case analysis of healthy control subjects, the latter factor might have accounted for performance differences. Selective interference on temporal recognition produced by a temporal classification task-when compared to spatial classification-was found in 3 subjects out of the subgroup of younger subjects $(n=5$; mean age $=30.8$ years $)$ and in only 1 subject out of the subgroup of older subjects ( $n=5$; mean age 41.4 years).
} 


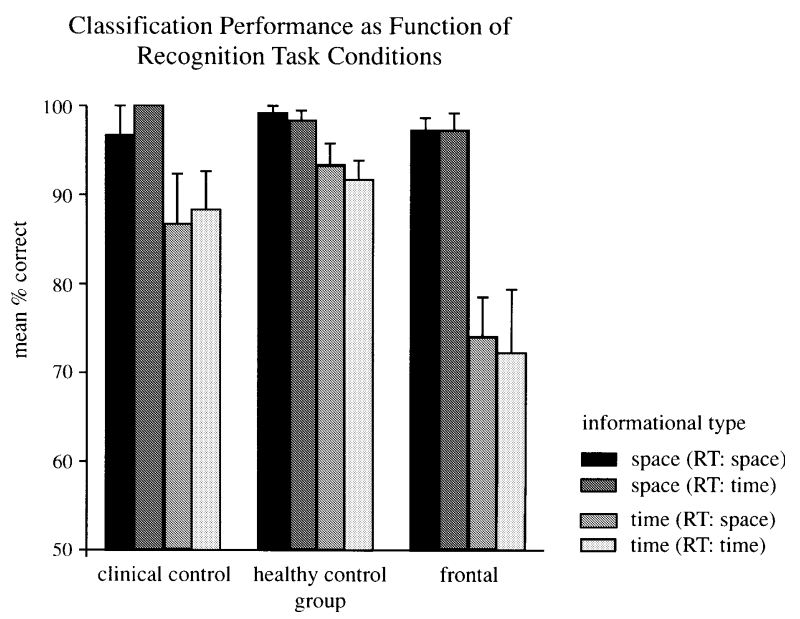

Fig. 7. Performance on spatial and temporal classification tasks as function of the spatial and temporal recognition tasks (RT) separately for clinical control group $(n=5)$, healthy control group $(n=10)$, and frontal group $(n=9)$. Bars represent mean accuracy and standard error of the means.

The difficulty level in both classification tasks was rather low as indicated by the performance of the healthy control group ( $>90 \%$ correct answers for both types of classification task). ANOVAs gave rise to significant effects of classification task, (frontal: $F(1,8)=24.66, p<.001$; healthy control: $F(1,9)=8.24, p<.02$ ) indicating higher performance levels in the spatial classification task for both the frontal and the healthy control group. However, neither effects of recognition task (frontal: $F(1,8)=.07, p=.79$ ); healthy control: $F(1,9)=1.33, p=.28)$, nor interactions were obtained (frontal: $F(1,8)=.16, p=.70$; healthy control: $F(1,9)=.13 p=.76$ ), reflecting that there were no proactive interference effects by the preceding recognition task study phases on the two different classification tasks.

The same result was obtained for the clinical control group. Wilcoxon's signedrank test revealed that there were no specific effects of spatial and temporal recognition task study phase on temporal $(z=-.45, p=.65)$ or spatial classification task $(z=-1.0, p=.32)$ performance.

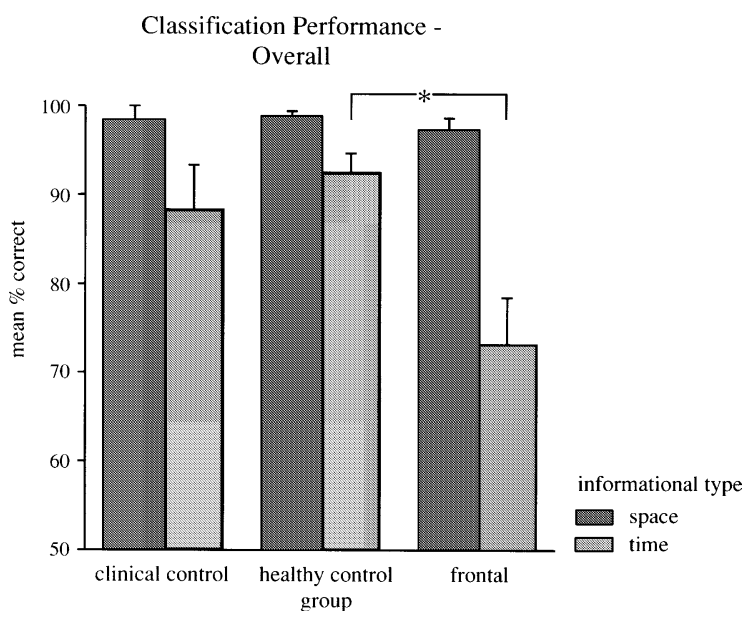

Fig. 8. Performance on spatial and temporal classification tasks pooled over both recognition task conditions separately for clinical control group $(n=5)$, healthy control group $(n=10)$, and frontal group $(n=9)$. Bars represent mean accuracy and standard error of the means. Asterisks denote significant differences from the performance of the healthy controls. 


\subsection{Classification performance-overall}

Fig. 8 shows the effects of cortical lesions on spatial and temporal classification tasks relative to healthy control subjects for both patient groups. For each classification task, data were collapsed over both recognition task conditions. Two-way repeated measure ANOVAs with between-subject factor group (two levels) and within-subject factor informational type (two levels) were performed for the quantification of differences between memory performance of frontal patients and healthy control subjects.

The effect of group $(F(1,17)=11.10, p<.004)$ was significant, indicating different performance levels for the frontal and the control group. As revealed by a significant effect of informational type $(F(1,17)=34.98, p<.001)$, classification task performance was different for spatial and temporal tasks. The key result was a highly significant interaction group $x$ informational type $(F(1,17)=12.08$, $p<.003$ ), showing that between-group differences varied as a function of information type. $t$ Tests for independent samples were used to further elucidate the latter interaction. Compared to healthy control subjects, subjects with frontal cortex damage were impaired in the temporal duration classification $(\Delta=-19.3 \%$; $t(10,54)=-3.36, p<.007)$, but not in the spatial location classification $(\Delta=$ $-1.5 \% ; t(17)=-1.03, p=.32)$.

In order to test for statistical differences between clinical and healthy control group, the Mann-Whitney $U$ test was used. Though patients with post-central lesions did perform on average $10 \%$ lower than healthy controls, the statistical analysis did not yield differences on the spatial $(U=27.5, p=.72)$ and temporal tasks $(U=22.5, p=.41)$. Thus, post-central cortical lesions did not lead to a significant degradation on either temporal or spatial classification.

\section{Discussion}

Based on findings indicating the importance of the frontal lobes for temporal information processing (Casini \& Ivry, 1999; Fuster, 1985; Mangels et al., 1998; Milner, Petrides, \& Smith, 1985; Nichelli et al., 1995; Shimamura et al., 1990) the aim of the present study was to examine more specifically the nature of the temporal processing deficits observed in frontal lobe patients. Given the involvement of the frontal lobes in executive monitoring functions on the one hand (Fuster, 1989; Owen et al., 1998; Petrides, 1996), and the observation that temporal processing deficits after frontal lobe lesions appear to depend on task characteristics on the other (Casini \& Ivry, 1999; Mangels, 1997; Mangels et al., 1998; Shimamura et al., 1990), we set out to test the hypothesis that impairments of duration processing after frontal lobe lesions reflect deficits in executive monitoring functions rather than a domain-specific deficit in encoding and maintenance of duration information in working memory.

Patients with frontodorsal lesions restricted to the superior or middle frontal gyri, patients with post-central lesions who served as clinical control subjects, and intact subjects were each tested on recognition and classification tasks. While recognition tests should allow for testing encoding and maintenance of information in working memory, classification tests placing additional executive demands were thought to test for the role of the frontal cortex in strategic monitoring. Since the tasks were embedded in a dual-task design, we shall discuss differential results obtained on recognition and classification tasks as well as interference effects among both of the tasks.

First of all, performance in all groups was overall worse on recognition tasks than on classification tasks. This might result from differences of the retention intervals in 
both task types. Remember that retention intervals in the recognition task amounted to more than $7 \mathrm{~s}$, whereas in the classification task the retention intervals were in the millisecond range.

\subsection{Recognition tasks}

The experiment showed that on recognition tests, where subjects had to indicate whether the study and the test stimuli were same or different in duration (temporal recognition) or in location (spatial recognition), the performance of the clinical control group did not differ significantly from the healthy control group. Frontal lobe patients were slightly impaired on both the temporal and the spatial condition. The statistical analysis revealed a mild trend without reaching significance. More important for the focus of this study, frontal lobe lesions did not affect performance on temporal and spatial tasks differentially.

The importance of the frontal lobes for working memory functions on delay tasks had been demonstrated earlier in lesion-, imaging- and electrophysiological studies on humans and non-human primates (Fuster, 1984; Fuster, 1991; Goldman-Rakic, 1994; Rypma, Prabhakaran, Desmond, Glover, \& Gabrieli, 1999; Vérin et al., 1993; Wilson et al., 1993). The result of a non-specific degradation of working memory functions after frontal lobe lesion is compatible with recent studies indicating poor performance after frontal lobe lesions on temporal as well as on non-temporal tasks (Casini \& Ivry, 1999; Mangels et al., 1998), especially when long ISIs were used. For example, Mangels et al. (1998) reported that frontal lobe patients' impairments on both duration estimation and frequency discrimination depend more on the length of time that the information must be maintained in working memory than on the type of information itself. Comparing performance on short- $(400 \mathrm{~ms})$ and long-range ISIs (4s), the frontal lobe patients showed impairments for both task dimensions on the long-range condition. Similarly, in the present study where recognition task ISIs were even longer $(>6 \mathrm{~s})$, deficits on duration recognition in the frontal group were non-selective to the temporal domain. Since there is evidence that performance on delay tasks depends on the duration of the retention interval, it is conceivable, although speculative, that the frontal group might not have been impaired at all on tasks using delay durations in the millisecond range. However, within the context of the current experimental design using long delays, the present data support the notion of a non-specific involvement of the frontal lobes in recognition, irrespective of the type of information.

\subsection{Recognition tasks under dual-tasks conditions}

On the recognition tasks under dual-task conditions, neither patient group showed a general performance drop when concurrent classification tasks had to be performed during the delay. For both the temporal and spatial recognition tasks, interference effects caused by concurrent classification were selective to the type of information to be processed during classification. Temporal recognition was selectively impaired by a temporal classification task but not by spatial classification, and spatial recognition was impaired by a spatial classification task but not by temporal classification. This result obtained for both patient groups is comparable to domain-specific interference effects reported for normal subjects (Hälbig et al., 1998). The finding that concurrent recognition and classification within the same trial per se did not lead to poorer performance indicates that our frontal lobe patients basically were able to cope with the strategic demands of the dual-task setting. Since interference only occurred when information of the same domain had to be processed on both tasks within a trial, it appears that the critical factors were not related to executive task demands but rather to concurrent memory load from the same informational domain. 
The classification tasks were restricted to the retention interval of the recognition task where the rehearsal component can be assumed dominant. As far as the prefrontal cortex is specifically involved in rehearsal processes mediating temporal duration memory, lesions should have lead to a disproportional degradation on temporal recognition when the underlying systems were taxed by concurrent temporal processing. This was not the case in our frontal lobe patients. In spite of the significantly higher level of difficulty of the temporal task, the size of selective interference in frontal patients was nearly symmetrical for both the temporal $(\Delta=21 \%)$ and the spatial domain $(\Delta=16 \%)$. This fact challenges the hypothesis of a privileged role of the prefrontal cortex as a storage site for temporal information, at least when compared to spatial memory.

\subsection{Classification tasks}

In contrast, on the classification tests, which had to be performed during the retention phase of the recognition task, only frontal patients were impaired, showing a marked selective performance drop $(\Delta=19.2 \%)$ compared to healthy controls when they had to classify if one of two different successively shown stimuli was longer in presentation duration (temporal classification) than the other. Like clinical controls the frontal patients performed within the range of healthy control subjects on the spatial classification test, i.e., when they had to classify if one of two successively shown stimuli was higher in position.

Although we had hypothesized greater impairment on temporal classification than on spatial classification for the frontal group, impairments on the spatial task were also expected. The level of difficulty for both the temporal and the spatial classification task can be considered as rather low. Nevertheless, all groups had relatively more difficulty on the temporal classification task (healthy control: $92 \%$ ) than on the spatial classification task (healthy control: $98 \%$ ). Thus, it is possible that the temporal duration task was more sensitive to frontal damage than the spatial classification task simply because of its higher degree of difficulty. This would be consistent with evidences that frontal lesions produce larger effects on demanding memory tests than on simpler ones (Wheeler, Stuss, \& Tulving, 1995). Yet the design of temporal and spatial tasks involved identical encoding and response principles. They differed only with regard to the type of information to be manipulated and-for demands resulting from the highly complex dual-task design-the delay. We cannot exclude that the latter factor might have accounted for the higher degree of sensitivity of the temporal task in frontal lobe patients. ${ }^{3}$ However, since several other lesion studies (e.g., Kesner et al., 1994; Milner et al., 1991; Shimamura et al., 1990) also reported differences in the difficulty of temporal and item memory tasks, it appears that temporal information processing in working memory generally taxes more cognitive resources than the processing of other types of information. Notwithstanding, the frontal group shows the largest relative within-group difference between performance on temporal and spatial classification.

\subsection{General discussion}

The marked selective degradation on temporal duration classification in frontal lobe patients contrasts with their only marginally impaired performance on temporal recognition. One explanation for the different performance on temporal classification and temporal recognition might be related to characteristics of the dual-task design. In contrast to the recognition task baseline condition, the classification tasks were to be performed during the retention phase of the recognition tasks. Although subjects were instructed to perform well on both tasks, it is possible that they focused primarily on the recognition task. In this case, attentional resources might have allowed 
sufficient performance on the recognition tasks but not for classification. In order to explain the full performance pattern by such a trade-off in resource allocation, we would have to assume that the different degrees of difficulty in the recognition tasks affected classification task performance differentially. Remember, the temporal recognition tasks had a significantly higher degree of difficulty than the spatial recognition tasks $(\Delta=14 \%, p<.0001)$. The possibility of an effect of recognition task on classification performance, however, was ruled out by statistical analysis. Performance on both the temporal and the spatial classification tasks was not differentially affected by the study phase of the preceding temporal and spatial recognition tasks. Different performance patterns in classification and recognition tasks are therefore unlikely to be attributed to problems of resource allocation during performance on the temporal classification tasks.

We assume rather that impairments on the temporal classification task with basically preserved performance on recognition tasks are caused by defective strategic monitoring. The importance of the prefrontal cortex for strategic monitoring functions is well known (Baddeley, 1986; Fuster, 1989). It has been shown that frontal lobe patients perform poorly on a large variety of working memory tests when the tests meet certain conditions related to executive monitoring (Fuster, 1989; Petrides, 1996; Shimamura et al., 1990). Even if in the present study recognition and classification tasks involve similar encoding, maintenance and retrieval operations, they differed regarding important task characteristics which theoretically might have affected performance differentially. For instance, in the recognition tasks, the ISI amounted to $6 \mathrm{~s}$ whereas ISIs in the classification tasks were rather short $(1500 \mathrm{~ms})$. A previous study using a somewhat different design (Mangels et al., 1998) revealed that performance on temporal duration and nontemporal tasks after frontal lobe lesions was degraded as a function of the length of the delay for which information has to be maintained, with impaired performance for longer delays in the range of $4 \mathrm{~s}$ and basically preserved performance in the ms range. In fact, in the present study, frontal lobe patients showed a marked selective impairment on the temporal classification task in spite of rather short ISIs $(1500 \mathrm{~ms})$, whereas performance on both the temporal and the spatial recognition task, where the ISI amounted to $6 \mathrm{~s}$, were only slightly impaired. Therefore, the marked selective performance drop on the temporal classification task cannot be explained by delay differences.

The differential outcome of classification and recognition tasks is much more likely to result from task differences more closely related to their strategic characteristics. Remember that, in comparison to the recognition tasks, the crucial input characteristics in classification tasks had to be associated with different object features (star, cross). Furthermore, in contrast to the recognition task where the reference stimulus always appeared first in order, the reference stimulus (star) in the classification task was shown in half of the trials temporally before the test stimulus (cross) and in the other half after the test stimulus. Thus, the classification task, demanded not only attentional resources to form internal representations of the crucial stimulus feature (i.e., temporal duration and spatial position, respectively) but, at the same time, sustained attention to the varying stimulus context characteristics. The view that these higher strategic demands of the classification tasks might account for the frontal patients' deficits on the temporal classification tasks is in accordance with recent findings showing impaired performance on temporal tasks also related to attentional demands (Casini \& Ivry, 1999; Mangels, 1997).

Since lesions in our frontal patients were restricted to the superior and middle frontal gyri, the interpretation of impaired temporal processing related to executive task demands is compatible with recent research suggesting that maintenance and monitoring components involve different frontal regions, with mid-dorsolateral 
portions of the prefrontal cortex mediating executive functions and mid-ventrolateral portions mediating maintenance (D’Esposito et al., 1998; Owen et al., 1998; Petrides, 1996; Postle et al., 1999). Nevertheless, the assumed organizational deficits seem to be selective to processes that are involved in temporal processing and not in non-temporal tasks (for a comparable result on temporal order tasks see Mangels, 1997). Indeed, there is evidence that acquisition, representation, and processing of temporal information in neural systems presumably differ from the processing of object or spatial stimulus features. For example, unlike stimulus dimensions like color and shape, temporal representations are acquired dynamically subserved by the use of effective encoding and monitoring strategies (Gilliland \& Martin, 1940; Kileen \& Weiss, 1987) and are thus specifically sensitive to attentional manipulations (Casini \& Ivry, 1999; Mangels et al., 1998; Zakay \& Block, 1996). The involvement of these strategic operations might have rendered the executive monitoring of duration information more sensitive to frontal lobe damage than the respective operations on spatial information.

In summary, the recognition task data of moderately impaired performance on both temporal duration and spatial location tasks after lesions of the superior and middle frontal gyri do give evidence for a non-specific involvement of these portions of the prefrontal cortex in maintenance of temporal information. This view is supported by the observation that selective interference on temporal recognition caused by temporal classification was not more pronounced than interference due to the respective spatial tasks. In contrast, on the classification tasks, frontal lobe patients showed a marked selective deficit in temporal information processing. Taken together, both results give further evidence for the hypothesis that impairments in temporal processing after frontal lobe lesions are secondary to deficits in strategic monitoring functions (Casini \& Ivry, 1999; Mangels, 1997; Shimamura et al., 1990). Our findings are compatible with the view that the functional significance of the prefrontal cortex for the processing of temporal information has to be described as rather process- than domain-specific. Accordingly, the prefrontal cortex appears to mediate executive monitoring of temporal information in the same manner as other types of information, with basic encoding and maintenance functions realized by other neural structures (Owen et al., 1998; Petrides, 1996). On the other hand, since strategic monitoring of temporal information after frontal lobe lesions seems to be more easily impaired than other informational domains, the contribution of prefrontal cortex to temporal processing is "domain-specific" in the sense that temporal representation formats require strategic monitoring operations that are specific to the temporal domain.

\section{Acknowledgments}

We are grateful to Prof. H.-J. Reulen, Department of Neurosurgery, LudwigMaximilians University, Munich, and to Prof. M. Brock and Dr. T. Höll, Department of Neurosurgery, Free University of Berlin, for the opportunity to study their patients; and A. Springer for assistance in data collection. We especially thank all the patients who so willingly participated in this experiment.

\section{References}

Baddeley, A. D. (1986). Working memory. Oxford: Oxford University Press.

Boltz, M. G. (1993). The remembering of auditory event durations. Journal of Experimental Psychology: Learning, Memory and Cognition, 18, 938-956.

Cabeza, R., Mangels, J. A., Nyberg, L., Habib, R., Houle, S., McIntosh, A. R., \& Tulving, E. (1997). Brain regions differentially involved in remembering what and when. Neuron, 19, 863-870. 
Casini, L., \& Ivry, R. B. (1999). Effects of divided attention on temporal processing in patients with lesions of the cerebellum or frontal lobe. Neuropsychology, 1, 10-21.

Casini, L., \& Macar, F. (1996). Prefrontal slow potentials in temporal compared to nontemporal tasks. Journal of Psychophysiology, 10, 252-264.

Church, R. M. (1989). Theories of timing behavior. In S. B. Klein \& R. R. Mowrer (Eds.), Contemporary learning theories: Instrumental conditioning theory and the impact of biological constraints on learning (pp. 41-71). Hillsdale, NJ: Erlbaum.

Coull, J. T., \& Nobre, A. C. (1998). When and where to pay attention: The neural systems for directing attention to spatial locations and to time intervals as revealed by PET and fMRI. Journal of Neuroscience, 18, 7426-7435.

D’Esposito, M., Aguirre, G. K., Zarahn, E., Ballard, D., Shin, R. K., \& Lease, J. (1998). Functional MRI studies of spatial and nonspatial working memory. Cognitive Brain Research, 7, 1-13.

Elbert, T., Ulrich, R., Rockstroh, B., \& Lutzenberger, W. (1991). The processing of temporal intervals reflected by CNV-like brain potentials. Psychophysiology, 28, 648-655.

Farah, M. J., Hammond, K. M., Levine, D. N., \& Calvanio, R. (1988). Visual and spatial mental imagery: Dissociable systems of representation. Cognitive Psychology, 20, 439-462.

Fuster, J. M. (1984). Behavioral physiology of the frontal cortex. Trends in Neuroscience, 7, 408-414.

Fuster, J. M. (1985). The prefrontal cortex, mediator of cross-temporal contingencies. Human Neurobiology, 4, 169-179.

Fuster, J. M. (1989). The prefrontal cortex. New York: Raven Press.

Fuster, J. M. (1991). Role of the prefrontal cortex in delay tasks: Evidence from reversible lesion and unit recording in the monkey. In H. S. Levin, H. M. Eisenberg, \& A. L. Benton (Eds.), Frontal lobe function and dysfunction (pp. 59-71). Oxford: Oxford University Press.

Gibbon, J., Church, R. M., \& Meck, W. H. (1984). Scalar timing in memory. Annals of the New York Academy of Sciences, 423, 52-77.

Gibbon, J., Malapani, C., Dale, Corby, L., \& Gallistel, C. R. (1997). Toward a neurobiology of temporal cognition: Advances and challenges. Current Opinions in Neurobiology, 7, 170-184.

Gilliland, A. R., \& Martin, R. (1940). Some factors in estimating short time intervals. Journal of Experimental Psychology, 27, 243-255.

Goldman-Rakic, P. S. (1994). The issue of memory in the study of prefrontal functions. In A. M. Theirry, J. Glowinski, P. S. Goldman-Rakic, \& Y. Christen (Eds.), Motor and cognitive functions of the prefrontal cortex (pp. 112-122). Heidelberg: Springer.

Grafman, J. (1989). Plans, actions, and mental sets: Managerial knowledge units in the frontal lobe. In E. Perecman (Ed.), Integrating theory and practice in clinical neuropsychology (pp. 93-138). Hillsdale, NJ: Erlbaum.

Hälbig, T. D., Mecklinger, A., Schriefers, H., \& Friederici, A. D. (1998). Double dissociation of processing temporal and spatial information in working memory. Neuropsychologia, 28, 803-813.

Hinton, S. C., Meck, W. H., \& MacFall, J. R. (1996). Peak-interval timing activates frontal-striatal loops. Neuroimage, 3, S224.

Ivry, R. B. (1996). The representation of temporal information in perception and motor control. Current Opinions in Neurobiology, 6, 851-857.

Ivry, R. B., \& Keele, S. W. (1989). Timing functions of the cerebellum. Journal of Cognitive Neuroscience, $1,136-152$.

Jueptner, M., Rijntjes, M., Weiller, C., Faiss, J. H., Timmann, D., Mueller, S. P., \& Diener, H. C. (1995). Localization of a cerebellar timing process using PET. Neurology, 45, 1540-1545.

Kesner, R. P., Hopkins, R. O., \& Fineman, B. (1994). Item and order dissociation in humans with prefrontal cortex damage. Neuropsychologia, 32, 881-891.

Kileen, P. R., \& Weiss, N. A. (1987). Optimal timing and the Weber function. Psychological Review, 94, 455-468.

Levy, R., \& Goldman-Rakic, P. S. (2000). Segregation of working memory functions within the dorsolateral prefrontal cortex. Experimental Brain Research, 133, 23-32.

Mangels, J. A. (1997). Strategic processing and memory for temporal order in patients with frontal lobe lesions. Neuropsychology, 11, 207-221.

Mangels, J. A., Ivry, R. B., \& Shimizu, N. (1998). Dissociable contributions of the prefrontal and neocerebellar cortex to time perception. Cognitive Brain Research, 7, 15-39.

McAndrews, M. P., \& Milner, B. (1991). The frontal cortex and memory for temporal order. Neuropsychologia, 29, 849-859.

Meck, W. H. (1996). Neuropharmacology of timing and time perception. Cognitive Brain Research, 3, 227-242.

Mecklinger, A., \& Pfeifer, E. (1996). Event-related potentials reveal topographical and temporal distinct neuronal activation patterns for spatial and object working memory. Cognitive Brain Research, 4, 211-224.

Miall, C. (1996). Models in neural timing. In M. A. Pastor \& J. Artieda (Eds.), Time, internal clocks, and movement (pp. 69-94). Amsterdam: Elsevier. 
Miller, R. R., \& Barnet, R. C. (1993). The role of time in elementary associations. Current Directions in Psychological Science, 2, 137-142.

Milner, B., Corsi, P., \& Leonard, G. (1991). Frontal-lobe contribution to recency judgments. Neuropsychologia, 29, 601-618.

Milner, B., Petrides, M., \& Smith, M. L. (1985). Frontal lobes and the temporal organization of memory. Human Neurobiology, 4, 137-142.

Newcombe, F., Ratcliff, G., \& Damasio, H. (1987). Dissociable visual and spatial impairments following right posterior cerebral lesions: Clinical, neuropsychological, and anatomical evidence. Neuropsychologia, 18, 149-161.

Nichelli, P. (1993). The neuropsychology of human temporal information processing. In F. Boller \& J. Grafman (Eds.), Handbook of neuropsychology (pp. 339-371). Amsterdam: Elsevier.

Nichelli, P., Clark, K., Hollnagel, C., \& Grafman, J. (1995). Duration processing after frontal lobe lesions. In J. Grafman, K. J. Holyoak, \& F. Boller (Eds.), Annals of the NY Academy of Science (pp. 183-190). New York, NY: Academy of Sciences.

Nichelli, P., Alway, D., \& Grafman, J. (1996). Perceptual timing in cerebellar degeneration. Neuropsychologia, 34, 863-871.

Niki, H., \& Watanabe, M. (1979). Prefrontal and cingulate unit activity during timing behavior in the monkey. Brain Research, 171, 213-224.

Owen, A. M., Stern, C. E., Look, R. B., Tracey, L., Rosen, B. R., \& Petrides, M. (1998). Functional organization of spatial and nonspatial working memory processing within the human lateral frontal cortex. Proceedings of the National Academy of Sciences USA, 95, 7721-7726.

Pastor, M. A., Artieda, J., Jahanshahi, M., \& Obeso, J. A. (1992). Time estimation and reproduction is abnormal in Parkinson's disease. Brain, 115, 211-225.

Petrides, M. (1996). Specialized systems for the processing of mnemonic information within the primate frontal cortex. Philosophical Transactions of the Royal Society of London: Series B, Biological Sciences, 351, 1455-1462.

Postle, B. R., Berger, J. S., \& D’Esposito, M. (1999). Functional neuroanatomical double dissociation of mnemonic and executive control processes contributing to working memory performance. Proceedings of the National Academy of Sciences, 96(22), 12959-12964.

Rammsayer, T. (1994). A cognitive-neuroscience approach for elucidation of mechanisms underlying temporal information processing. International Neuroscience, 77, 61-76.

Rammsayer, T., \& Lima, S. (1990). Duration discrimination of filled and empty auditory intervals: Cognitive and perceptual factors. Perception and Psychophysics, 50, 565-574.

Ruchkin, D. S., Johnson, J. R., Grafman, J., Canoune, H., \& Ritter, W. (1997). Multiple visuospatial working memory buffers: Evidence from spatiotemporal patterns of brain activity. Neuropsychologia, 35, 195-209.

Rypma, B., Prabhakaran, V., Desmond, J. E., Glover, G. H., \& Gabrieli, J. D. E. (1999). Load-dependent roles of frontal brain regions in the maintenance of working memory. NeuroImage, 9, 216-225.

Schubotz, R., \& Friederici, A. D. (1997). Electrophysiological correlates of temporal and spatial information processing. Neuroreport, 8, 1981-1986.

Shimamura, A. P., Janowsky, J. S., \& Squire, L. R. (1990). Memory for the temporal order of events in patients with frontal lobe lesions and amnesic patients. Neuropsychologia, 28, 803-813.

Shimamura, A. P., Jernigan, T. L., \& Squire, L. R. (1988). Korsakoff's syndrome: Radiological (CT) findings and neuropsychological correlates. Journal of Neuroscience, 8, 4400-4410.

Smith, E. E., Jonides, J., Koeppe, R. A., Awh, E., Schumacher, E. H., \& Minoshima, S. (1995). Spatial versus object working memory: PET investigations. Journal of Cognitive Neuroscience, 7, 337-356.

Squire, L. R. (1982). Comparisons between forms of amnesia: Some deficits are unique to Korsakoff's syndrome. Journal of Experimental Psychology: Learning, Memory and Cognition, 8, 560-571.

Tracey, J. I., Faro, S. H., Mohamed, F. B., Pinsk, M., \& Pinus, A. (2000). Functional localization of a "Time Keeper" function separate from attentional resources and task strategy. NeuroImage, 11, $228-242$.

Treisman, M., Faulkner, A., Naish, P. L. N., \& Brogan, D. (1990). The internal clock: Evidence for a temporal oscillator underlying time perception with some estimates of its characteristic frequency. Perception, 19, 705-743.

Tresch, M. C., Sinnamon, H. M., \& Seamon, J. G. (1993). Double dissociation of spatial and object visual memory: Evidence from selective interference in intact human subjects. Neuropsychologia, 31, 211-219.

Ungerleider, L. G., \& Mishkin, M. (1982). Two cortical visual systems. In D. J. Ingle, M. A. Goodale, \& R. J. W. Mansfield (Eds.), Analysis of visual behaviour (pp. 549-587). Cambridge, MA: MIT Press.

Vérin, M., Partiot, A., Pillon, B., Malapani, C., Agid, Y., \& Dubois, B. (1993). Delayed response tasks and prefrontal lesions in man-Evidence for self-generated patterns of behaviour with poor environmental modulation. Neuropsychologia, 12, 1379-1397.

Wheeler, M. A., Stuss, D. T., \& Tulving, E. (1995). Frontal lobe damage produces episodic memory impairment. Journal of the International Neuropsychological Society, 1, 525-536. 
Wilson, F. A. W., O'Scalaidhe, S. P., \& Goldman-Rakic, P. S. (1993). Dissociation of object and spatial processing-domains in primate prefrontal cortex. Science, 260, 1955-1985.

Zakay, D., \& Block, R. (1996). The role of attention in time estimation processes. In M. A. Pastor \& J. Artieda (Eds.), Time, internal clocks, and movement (pp. 143-164). Amsterdam: Elsevier. 